\title{
Effect of Classical Hata Yoga Training On Executive Function in Healthy Adults
}

\author{
Dr. Vijaya kumar $\mathrm{PS}^{1 *}$, Sahana $\mathrm{AU}^{2}$ \\ ${ }^{1}$ Associate professor, BAMS, MD (Yoga \& rehab), Msc (psy), ${ }^{2}$ Clinical Psychologist Msc (Clinical psychology) Department of Astanga yoga Lakulish \\ Yoga University "Lotus view" opp. Nirma University, S.G. Highway, Chharodi, Ahmedabad - 382481 Gujarat, India
}

DOI: $10.36348 /$ SIJTCM.2019.v02i08.001 $\quad$ | Received: 15.10.2019| Accepted: 22.10.2019| Published: 30.10 .2019

*Corresponding author: Dr. Vijayakumar PS

Abstract

Background: Executive Function is a higher order of cognitive processes, mild cognitive impairment is associated with normal ageing, the world is aging rapidly through "demographic transition" resulted in early cognitive decline, there is a connection between cognition components with mental health disorders. Mind-body techniques such as yoga have shown to improve cognitive functions and enhancing psychological wellbeing. The objective of this study was to determine whether classical hata yoga training enhances the executive function resulting in improved cognitive functions. Material and Methods: Thirty four participants who had enrolled in a forty five days classical hata yoga training program. Their ages ranged between 18 and 50 years (group average \pm S.D., 31.4 \pm 9.3 years, both genders). Those who have any chronic illness and mental illness, and those who are not willing to participate were excluded. Classical hata yoga training is given for 45 days, 3hours/day. At baseline and following forty five days, all participants completed DLST and SLCT. Results: Classical hata yoga training program showed significant change in DLST scores, increase $(\mathrm{P}-\mathrm{value}<0.000)$ in total attempted score, significant increase $(\mathrm{P}-\mathrm{value}<0.000)$ in net score, significant reduction in wrongly attempted score (P-value $<0.004)$. Significant change in SLCT scores, significant increase $(\mathrm{P}$-value $<0.000)$ in total attempted score, similarly, significant increase $(\mathrm{P}$-value $<.000)$ in net score, significant reduction in wrongly attempted score $(\mathrm{P}$ value $<0.000$ ). Conclusions: The forty five days classical hata yoga training program was successful in enhancing the executive function among healthy adults.

Keywords: Executive function (EF), Classical hata yoga, Cognitive functions, SLCT, DLST.

Copyright @ 2019: This is an open-access article distributed under the terms of the Creative Commons Attribution license which permits unrestricted use, distribution, and reproduction in any medium for non-commercial use (NonCommercial, or CC-BY-NC) provided the original author and source are credited.

\section{INTRODUCTION}

Executive Function (EF) is a higher order of cognitive processes, such as cognitive flexibility, working memory, and inhibition control which allow individuals to engage in planning, to be conscious and goal-directed problem solving [1]. EF depends mainly on intact frontal lobe. The frontal lobe controls various body functions and grows through puberty and adult life. Some of its functions include emotion, attention, working memory, abstract reasoning, planning, selfregulation of emotions, and start of goal directed behaviors [2]. EF includes different cognitive abilities such as, attention, memory, and self-regulation of emotions, behavioral inhibition and other high order activities [3]. Mild cognitive impairment is associated with normal ageing; the world is aging rapidly through "demographic transition." In 2011, 11\% global [4] and $8 \%$ Indian population is $>60$ years and would reach $19 \%$ by 2050 [5]. Cognitive decline is increasing at an alarming rate in developing countries such as India.
Earlier findings suggest that brain exposure to higher cortisol concentrations contribute to cognitive deficits as we age. The executive function defects can result in multiple poor consequences, such as depression, attention disorders, and aggression [6]. The cognitive training could reduce the psychological distress and depression level, plus this type of training improved the cognitive function and working memory. This indicated that there is a connection between cognition components with mental health disorders [7]. Yoga is an ancient Indian mind body practice and way of life that includes the practice of specific postures, regulated breathing and meditation [8]. Meditation (intrinsic yoga techniques called Dharana, Dhyana and Samadhi) has been described as training in awareness, produces definite changes in perception, attention and cognition [9]. It has been shown that processing of sensory information at the thalamic level is facilitated during the practice of pranayama (breathing exercises)[10] and meditation[11], this improvement was believed to be 
due to improved eye hand coordination, attention, concentration and relaxation. With these promising benefits of yoga, we could hypothesise that yoga may decrease the cognitive dysfunction. Previous studies on sustain attention suggests that reduced anxiety can improve the performance on tasks [12] and yoga's anxiety reducing effects [13] could also have facilitated this. Physical postures (Asana), voluntary regulation of breathing (Pranayama), maintaining silence, and visual focusing exercises (Trataka) improve attention span in school children $[14,15]$. However, yoga also appears to offer potential psychological benefits through the inclusion of mindfulness training, involving the practice of meditation as well as the dynamic combination of proprioceptive and interoceptive awareness [16]. We hypothesized that classical hata yoga training would result in improved EF performance on outcome measures assessing visual scanning, mental flexibility, sustained attention, psychomotor speed, and speed of information processing. It was further hypothesized that improvements in EF would be associated with improvements in psychological wellbeing, yoga practice leads to substantial neurobiological and psychophysiological alterations. Some studies indicate that yoga has been found to enhance resting state activation across multiple cortico-striatals neuronal loops [17], a system thought to sub serve executive functions [18], and for which alterations have been associated with a host of neuropsychiatric disorders [19]. The current study examined whether forty five days of classical hata yoga training would result in improvement in EF.

\section{MATERIAL AND METHODS}

Thirty four participants who had enrolled in a forty five days classical hata yoga training program. Their ages ranged between 18 and 50 years (group average \pm S.D., $31.4 \pm 9.3$ years; 28 females and 6 males). We have fully explained the potential risks and benefits in the study before written informed consent was provided by participants, the study was approved by the ethics committee of the institution, Lakulish yoga university, located in Ahmadabad, Gujarat, India. The selection criteria included: Subjects who were healthy, age between-18-50 yrs, Education: $12^{\text {th }}$ standard and above and those knowing English, willing to participate were included. Those having neurological and psychiatric disorders (based on case history), those who have practiced Yoga for the last 3 months and those who have major eyesight problems (in vision) were excluded from the study. In this study we adopted a convenient sampling method to recruit the subjects who were undergoing forty five days Classical Hata yoga training program at Lakulish yoga university, Ahmadabad, Both genders, Age group 18 to 50 years, a single group pre-post design was used. The 45 days study was successfully completed by 34 participants.

\section{Design and setting}

The trial was a single group, pre-post trial. Participants were assessed on day 1 and day 45 of the forty five days classical hata yoga training program, when the assessment was completed, respondents were appreciated for their time and cooperation. The training program was held in a non-residential Lakulish yoga university, located in Ahmadabad, Gujarat, India.

\section{Intervention}

The forty five days classical hata yoga training program: a yoga teacher training program consisted of two sessions each day. The first session was between 08:30 hours and 11:30 hours for practice and the second session was between 12:00 hours and 13:30 hours for theory. In a day participants practiced shithilikarana vyayamas (loosening practices) followed by yogasanas and relaxation techniques with pranayama practices. The concepts used to develop a specific module of teaching for training program were taken from the classical hata yoga scripture of Lakulish yoga tradition [20], one of the oldest schools of hata yoga in India. Yoga is defined as mastery over the modifications of mind (Chitta Vritti Nirodhah-definition of yoga by Patanjali). It helps to remove the unnecessary surges of neuromuscular activation resulting from heightened stress responses that may contribute to aging.

\section{Assessments}

SLCT -Cancellation tests require visual selectivity and a repetitive motor response. A six-letter cancellation test was administered to assess functions such as selective and focused attention, visual scanning, and the activation and inhibition of rapid responses. The six letter cancellation test has been used in similar type of design on Indian population[21].The six letter cancellation task worksheet consists of an array of random alphabets, A-Z, in 14 rows and 22 columns. Participants were asked to sit with the worksheet distributed to each one. The instructions are given asking them to cancel as many target digits as possible in the specified time. They are asked to cancel as their wish whether horizontally, vertically, or selecting a particular letter one at a time randomly in the row. Finally, after knowing the test instructions they are asked to start the test, each test was conducted for 90 seconds on a standard stopwatch.

DLST- Digit letter substitution test contains flexibility at mind level, visual scanning, attention and psychomotor speed of processing information. It is used with same type of design on Indian population [21]. DLST worksheet consists a row of random digits, 1-9, in 8 rows and 12 columns. The coding sheet contains instructions about the test with example of substituting a specific letter for specific digit 1-9, the same code is applicable to entire test. Subjects were instructed to make their choice of letter substitution process, whether horizontally, vertically, or selecting a particular digit 
randomly in the row one at a time. In given time of 90 seconds' substitute as many target digits as possible.

\section{DATA ANALYSIS}

The data taken on the last day and on the first day of the classical hata yoga training program were compared with t-test and wilcoxon signed rank test for paired using SPSS version 16.0.

\section{RESULTS}

A total of 34 subjects were participated in the study 34 subjects completed the study, which was conducted at the end of forty five days training program; Mean values and standard deviation for total scores, wrong substitutions, and net scores of six-letter cancellation task and digit-letter substitution task.

\section{SLCT: Six Letter Cancellation Test}

After 45 days of classical hata yoga training program it showed that $22 \%$ significant increase ( $\mathrm{P}<$ 0.000 , paired samples t-test) in total attempted (TA) score on SLCT. Similarly, there was $27 \%$ significant increase $(\mathrm{P}=0.000$, paired samples t-test) in NS. However, there was $93 \%$ decrease in wrongly attempted (WA) score which was highly significant (P $=0.000$, Wilcoxon Signed Ranks Test) [Table 1].

Table-1: SLCT Changes after Classical hata yoga training program

\begin{tabular}{|c|c|c|c|c|}
\hline \multirow[t]{2}{*}{ SLCT scores } & \multicolumn{2}{|c|}{ Classical hata yoga training } & (4) \% increase & P-value \\
\hline & Before & After & ( $\downarrow$ ) \% increase & \\
\hline TA & $34.44 \pm 10.43$ & $41.85 \pm 10.99$ & $4 \quad 22$ & $0.000 * * *$ \\
\hline WA & $1.64 \pm 1.73$ & $0.11 \pm 0.40$ & 193 & $0.000 * * *$ \\
\hline NS & $32.79 \pm 10.60$ & $41.73 \pm 10.94$ & 427 & $0.000 * * *$ \\
\hline
\end{tabular}

*significant at $\mathrm{P}<0.05$, ** significant at $\mathrm{P}<0.01$, ***significant at $\mathrm{P}<0.00$ (paired sample test and Wilcoxon Signed Ranks Test) SLCT: Six Letter Cancellation Test, TA: Total attempted, WA: Wrongly attempted, NS: Net scores

\section{DLST: Digit letter substitution test}

After 45 days of classical hata yoga training program it showed that $10 \%$ significant increase $(\mathrm{P}<$ 0.000 , paired samples t-test) in total attempted (TA) score on DLST. Similarly, there was $11 \%$ significant increase $(\mathrm{P}=0.000$, paired samples t-test $)$ in NS. However, there was $86 \%$ decrease in wrongly attempted (WA) score which was highly significant (P $=0.004$, Wilcoxon Signed Ranks Test) [Table 2].

Table-2: DLST Changes after Classical hata yoga training program

\begin{tabular}{|l|l|l|l|l|}
\hline \multirow{2}{*}{ DLST scores } & Classical hata yoga training & $(\mathbf{A})$ \% increase & \multirow{2}{*}{ P- value } \\
\cline { 2 - 4 } & Before & After & $(\downarrow)$ \% increase & \\
\hline TA & $58.29 \pm 11.74$ & $64.32 \pm 12.13$ & $\uparrow 10$ & $0.000^{* * *}$ \\
\hline WA & $0.76 \pm 1.15$ & $0.11 \pm 0.32$ & $\downarrow 86$ & $0.004^{* *}$ \\
\hline NS & $57.52 \pm 11.96$ & $63.82 \pm 12.32$ & $\uparrow 11$ & $0.000^{* * *}$ \\
\hline
\end{tabular}

*significant at $\mathrm{P}<0.05$, ** significant at $\mathrm{P}<0.01$, ***significant at $\mathrm{P}<0.00$ (paired sample test and Wilcoxon Signed Ranks Test) DLST: Digit letter substitution test, TA: Total attempted, WA: Wrongly attempted, NS: Net scores

\section{DISCUSSION}

The purpose of this study was to investigate the efficacy of forty five days classical hata yoga intervention for improving cognition, particularly executive function in a healthy adult population. Completion of this program was associated with overall significant improvement in measures of executive functions. Substitution tests are essentially speeddependent tasks that require the subject to match particular signs - symbols, digits, or letters - to other signs within a specified time period. Substitution tasks involve visual scanning, mental flexibility, sustained attention, psychomotor speed, and speed of information processing [22, 23]. Classical hata yoga training has emerged as a preventive modality for cognitive decline By enhancing the executive functions, yoga found to reduce cognitive deficits, with the expectation that improvement of cognition would result in improvement of psychosocial functioning [24]. Two recent studies have demonstrated 12 weeks of yoga sessions were positively associated with acute increase in thalamic GABA levels, improvement in mood and anxiety scales, and reduction in depressive symptoms [25]. Improvement of psychosocial functioning not only requires sustain attention, but also cognitive flexibility, working memory, and inhibition control. Decrease in total time taken and error scores in SLCT and DLST following Classical hata yoga training suggest improvement in Executive Function (EF). The mechanism underlying the improvement of EF may be related to the fact that hata yoga involves the practice of physical postures in conjunction with awareness of the breath to help develop mental focus and to connect the mind, body, and spirit [26]. Yoga postures are performed with a gap in between, provide relaxation to mind and body, and then ultimately enhances cognition. Previous studies on yoga techniques which consisted of sequence of yoga postures interspersed with relaxation 
techniques, found improvement in selective attention [27], and inhibition of the cortical region[28].Classical hata yoga requires focused effort in moving through the poses, controlling the body, and breathing at a steady rate, this focus on one's body and mind during the practices, breathing and meditation exercises are practiced to calm and focus the mind and develop greater self-awareness [29] high frequency yoga breathing practice (Kapalabhati) enhances blood flow to pre frontal cortex,[30] Pre-frontal cortex is associated with memory, attention, and $\mathrm{EF}[31,32]$. Kapalabhati and Bhastrika Pranayama had influence on auditory working memory, and central neural processing and sensory-motor performance [33], Bhramari Pranayama may enhance inhibition response and cognitive control in healthy participants [34]. The present study is the first attempt to evaluate the effect of classical hata yoga training on healthy adults in a non-residential set up; present study suggests that classical hata yoga training could help to prevent the early cognitive decline. Limitation of the present study was that it did not have a control group.

\section{CONCLUSIONS}

The forty five days classical hata yoga training program was successful in improving sustain attention, working memory capacity among healthy adults, a longer follow-up period will enable researchers to thoroughly examine neuro-cognitive changes. Although this was limited by small sample size, lack of heterogeneous population, these findings suggests rigorous systematic approaches and advanced imaging techniques to examine classical hata yoga training as a means to enhance executive function and prevent early cognitive decline.

\section{ACKNOWLEDGMENT}

We would like to thank all the subjects for participating in this research study and also thank the training program in-charge Mr.Sachin patel and team for their constant support to complete the study successfully.

\section{REFERENCES}

1. Diamond, A. (2013). Executive functions. Annu Rev Psychol, 64, 135-168.

2. Black, D.S., Semple, R.J., Pokhrel, P., Grenard, J.L. (2011). Component Processes of Executive Function-Mindfulness, Self-control, and Working Memory-and Their Relationships with Mental and Behavioral Health. Mindfulness, 2(3), 179-185.

3. Suchy, Y. (2009). Executive functioning: overview, assessment, and research issues for nonneuropsychologists. Ann Behav Med, 37(2), 106116.

4. United Nations Population Division (UN), World Population Prospects: The 2010 Revision. New York: United Nations; 2011. Available from: http://www.esa.un.org/unpd/wpp/index.htm.
5. Agrawal, A. (2013). Disability among the elder population of India: A public health concern. J Med Soc, 30, 15-9.

6. Greenberg, M.T. (2006). Promoting resilience in children and youth: preventive interventions and their interface with neuroscience. Annals of the New York Academy of Sciences, 1094, 139-150.

7. Preiss, M., Shatil, E., Cermáková, R., Cimermanová, D., \& Ram, I. (2013). Personalized cognitive training in unipolar and bipolar disorder: a study of cognitive functioning. Frontiers in human neuroscience, 7, 108.

8. Taimini, I.K. (1961). The Science of Yoga. Madras, India: The Theosophical Publishing House.

9. Brown, D.P. (1977). A model for the levels of concentrative meditation. Int J Clin Exp Hypn, 25, 236-73.

10. Telles, S., Joseph, C., Venkatesh, S., Desiraju, T. (1992). Alteration of auditory middle latency evoked potentials during yogic consciously regulated breathing and attentive state of mind. Int J Psychophysiol, 14, 189-98.

11. Telles, S., Desiraju, T. (1993). Autonomic changes in Brahmakumaris raja yoga meditation. Int $\mathrm{J}$ Psychophysiol, 15, 147-52.

12. Saltz, E. (1970). Manifest anxiety: Have we missed the data? Psychol Rev, 77, 568-73.

13. Wallace, R.K., Benson, H., Wilson, A.F. (1971). A wakeful hypometabolic physiologic state. Am J Physiol, 221, 795-9.

14. Sarang, S.P., Telles, S. (2007). Immediate effect of two yoga-based relaxation techniques on performance in a letter-cancellation task. Percept Mot Skills, 105, 379-85.

15. Telles, S., Raghuraj, P., Maharana, S., Nagendra, H.R. (2007). Immediate effect of three yoga breathing techniques on performance on a lettercancellation task. Percept Mot Skills, 104, 1289-96.

16. Mehling, W.E., Price, C., Daubenmier, J.J., Acree, M., Bartmess, E., \& Stewart, A. (2012). The Multidimensional Assessment of Interoceptive Awareness (MAIA). PloS one, 7(11), e48230.

17. Gard, T., Taquet, M., Dixit, R., Holzel, BK., Dickerson, B.C., \& Lazar, S.W. (2015). Greater widespread functional connectivity of the caudate in older adults who practice kripalu yoga and vipassana meditation than in controls. Frontiers in human neuroscience, 9, 137.

18. Leh, S.E., Petrides, M., Strafella, A.P. (2010). The Neural Circuitry of Executive Functions in Healthy Subjects and Parkinson 's disease. Neuropsychopharmacology, 35(1), 70-85.

19. Shepherd, G.M.G. (2013). Corticostriatal connectivity and its role in disease. Nature reviews Neuroscience, 14(4), 278-91.

20. Swami Rajarshri Muni. (2007). Classical hatha yoga. Vadodara: Life mission publication.

21. Natu, M.V., Agarawal, A.K. (1997). Testing of stimulant effects of coffee on the psychomotor 
performance: an exercise in clinical pharmacology. Indian Journal of Pharmacology, 29(1), 11-14.

22. Lezak, M.D. (1995). Neuropsychological Assessment 3rd ed, New York: Oxford U.P.

23. Van Hoof, J.J., Jogems-Kosterman, B.J., Sabbe, B.G., Zitman, F.G., \& Hulstijn, W. (1998). Differentiation of cognitive and motor slowing in the Digit Symbol Test (DST): Differences between depression and schizophrenia. J Psychiatr Res, 32, 99-103.

24. Balaji, P.A., Varne, S.R., Ali, S.S. (2012). Physiological effects of yogic practices and transcendental meditation in health and disease. $\mathrm{N}$ Am J Med Sci, 4(10), 442-448.

25. Streeter, C., Gerbarg, P., \& Saper, R. (2012). P01.31. Yoga therapy associated with increased brain GABA levels and decreased depressive symptoms in subjects with major depressive disorder: a pilot study. BMC Complementary and Alternative Medicine, 12(Suppl 1), P31.

26. Roland, K. P., Jakobi, J. M., Jones, G.R. (2011). Does yoga engender fitness in older adults? A critical review. J Aging Phys Act, 19, 62-79.

27. Sarang, S. P., \& Telles, S. (2007). Immediate Effect of Two Yoga-Based Relaxation Techniques on Performance in a Letter-Cancellation Task. Perceptual and Motor Skills, 105(2), 379-385.

28. Subramanya, P., \& Telles, S. (2009). Changes in midlatency auditory evoked potentials following two yoga-based relaxation techniques Clin EEG Neurosci, 40,190-195.

29. Morone, N.E., \& Greco, C.M. (2007). Mind-body interventions for chronic pain in older adults: a structured review. Pain Med, 8, 359-375.

30. Bhargav, H., Nagendra, H.R., Gangadhar, B.N., Nagarathna, R.(2014). Frontal hemodynamic responses to high frequency yoga breathing in schizophrenia: a functional near-infrared spectroscopy study Front Psychiatry, 5, 29.

31. Gray, J.R., Braver, T.S., Raichle, M. E. (2002). Integration of emotion and cognition in the lateral prefrontal cortex. Proc Natl Acad Sci, 99, 41154120.

32. West, R.L. (1996). An application of prefrontal cortex function theory to cognitive aging Psychol Bull, 120, 272-292.

33. Sharma, V. K., M, R., S, V., Subramanian, S. K., Bhavanani, A. B., Madanmohan, Thangavel, D. (2014). Effect of fast and slow pranayama practice on cognitive functions in healthy volunteers. Journal of clinical and diagnostic research: JCDR, 8(1), 10-13.

34. Rajesh, S.K., Ilavarasu, J.V., Srinivasan, T. M. (2014). Effect of Bhramari Pranayama on response inhibition: evidence from the stop signal task Int $\mathbf{J}$ Yoga, 7,138-141. 